\title{
Individual differences in spatial pattern separation performance associated with healthy aging in humans
}

\author{
Shauna M. Stark, Michael A. Yassa, and Craig E.L. Stark ${ }^{1}$ \\ Center for the Neurobiology of Learning and Memory, Department of Neurobiology and Behavior, University of California, Irvine, \\ Irvine, California 92697, USA
}

\begin{abstract}
Rodent studies have suggested that "pattern separation," the ability to distinguish among similar experiences, is diminished in a subset of aged rats. We extended these findings to the human using a task designed to assess spatial pattern separation behavior (determining at time of test whether pairs of pictures shown during the study were in the same spatial locations). Using a standardized test of word recall to divide healthy aged adults into impaired and unimpaired groups relative to young performance, we demonstrate that aged impaired adults are biased away from pattern separation and toward pattern completion, consistent with the rodent studies.
\end{abstract}

Memory impairment is a common complaint among aging individuals, yet the variability within the aging population is great in both rats (Gallagher et al. 2006; Robitsek et al. 2008) and humans (Hilborn et al. 2009). A rodent model of aging (Gallagher et al. 2006; Wilson et al. 2006) has demonstrated that $\sim 50 \%$ of healthy rats qualify as cognitively "impaired" by scoring outside the range of the young performance in a standard protocol (Gallagher et al. 1993). The other half, the "unimpaired" rats, perform on par with young adults, demonstrating a natural degree of variability in cognitive aging. In this study, we sought to capitalize on the variability observed in the aging of both rats and humans in a study of spatial pattern separation.

One source of variability in memory performance is hypothesized to be tied to changes in the input to the dentate gyrus (DG), which has been shown in the rat to be affected by the aging process. Smith et al. (2000) reported a selective impairment in layer II entorhinal input into the DG and CA3 regions of the hippocampus in rats with cognitive impairment. Similarly, the number of synapses in the outer receiving layer of DG was reduced in autopsied aged brains and correlated with earlier performance on a delayed recall task (Scheff et al. 2006). Finally, in a human imaging study, Small et al. (2002) observed that $60 \%$ of their aging sample demonstrated diminished MRI signal in the hippocampal region (including the DG) and also had a greater decline in memory performance. These findings support the notion that changes in the DG associated with aging may affect memory performance.

The DG may be particularly important for the computations that underlie pattern separation (Treves and Rolls 1994; McClelland et al. 1995; Norman and O'Reilly 2003). "Pattern separation" refers to the process by which similar inputs are stored as distinct, nonoverlapping representations. In contrast, "pattern completion" refers to the process by which an existing representation can be reinstated by the presentation of a partial or degraded cue. Numerous studies in the rodent have identified the importance of the DG for pattern separation using electrophysiological methods (Leutgeb et al. 2004, 2005, 2007; Leutgeb and Leutgeb 2007), immediate early gene expression (Vazdarjanova and Guzowski 2004), lesions (Lee et al. 2005; Gilbert and Kesner 2006; Goodrich-Hunsaker et al. 2008), and even genetic manipu-

\footnotetext{
'Corresponding author.
}

E-mail cestark@uci.edu; fax (949) 824-2447.

Article is online at http://www.learnmem.org/cgi/doi/10.1101//m.1768110. lations (Cravens et al. 2006; Kubik et al. 2007; McHugh et al. 2008). Human neuroimaging has also recently identified activity in the DG (and CA3 regions of the hippocampus) in an object pattern separation task (Kirwan and Stark 2007; Bakker et al. 2008).

Given the importance of the DG in pattern separation and its vulnerability to changes that occur with aging, studies have begun to examine pattern separation in older adults. Our laboratory has designed a task to examine object-based pattern separation performance in humans (Kirwan and Stark 2007). In this task, pictures of objects were presented either once or repeatedly throughout the task. Critically, some of the items presented were lures that were similar but not identical to previously shown items. The overlapping features of the lures more heavily engaged pattern separation processes. In young adults, functional magnetic resonance imaging (fMRI) activity in the DG was sensitive to the lures, indicating a role in pattern separation processes in both an explicit (Kirwan and Stark 2007) and implicit (Bakker et al. 2008) version of this task. Toner et al. (2009) used the explicit version of this task to demonstrate that older adults showed a greater tendency to identify lures as "old" (repeated) relative to young adults. These findings were also recently replicated in our laboratory (Yassa et al., in press), with the additional demonstration that older adults exhibit greater fMRI CA3/DG activity for the lures during both encoding and retrieval.

Since object-based pattern separation appears to be modulated by the DG in humans, we wondered if these findings could be extended to spatial pattern separation. Rodent studies have demonstrated that the DG has a particular role in spatial pattern separation (Gilbert et al. 2001; Kesner et al. 2004). Specifically, Hunsaker et al. (2008) placed rats with localized DG lesions in an environment with two objects spaced $60 \mathrm{~cm}$ apart. When the animals were later placed in the same environment with the same objects now placed $40 \mathrm{~cm}$ apart, DG-lesioned animals (unlike control animals) did not re-explore the objects or environment. These data suggest that the DG-lesioned rats were not able to discriminate between the training and test environments. That is, they were impaired in spatial pattern separation. Since converging evidence suggests that one feature of the aging process can be characterized as a DG knockdown, we modified this task design for humans to test spatial pattern separation performance in older adults. While the Hunsaker et al. (2008) task emphasized the distance between the two objects as the source of interference creating a greater need for pattern separation, the paradigm presented here moves an object in any direction, changing both the distance 
and the angle (i.e., changing more of the spatial relations). We posit that this amount of movement (close, medium, or far) may place similar demands on spatial pattern separation processes as in the rodent task.

The present study included 20 young adults (mean age 19.9 yr, range 18-27 yr) and 30 aged adults (mean age 70.4 yr, range $59-80 \mathrm{yr}$ ). Aged adults completed a battery of standardized neuropsychological tests, including the Mini-Mental State Exam (Folstein et al. 1975), Rey Auditory-Verbal Learning Task (RAVLT) (Rey 1941), Digit Span, Vocabulary, and Matrices subtests from the Wechsler Adult Intelligence Scale III (Wechsler 1997). The Vocabulary and Matrices scores were entered into a weighted formula along with age, gender, and education to derive estimated IQ scores (Schoenberg et al. 2003). All aged participants scored within the normal age-adjusted ranges on these measures and were cognitively intact. Younger adults also completed the RAVLT and scored within the normal age-adjusted range. These data are presented in Table 1.

The Spatial Pair Distance (SPD) task consisted of 10 study and test blocks for a total of 100 test pairs. Participants studied 10 unique pairs of pictures per block and were then tested on whether each of the 10 pairs was in the same or different locations compared to the study session. During the study session, participants viewed pairs of pictures for 2 sec each and were told to "try to remember the location of the pictures." During the test session, participants were told to indicate (with a key press) whether the pictures were in the same location as before or whether one of the pictures was in a different location. They were not told which of the two pictures might change position and the test was selfpaced. Critically, for the different trials, only one picture of the pair changed location. It could be moved a small amount (close; $10 \%-20 \%$ of the screen; $2.64^{\circ}-5.72^{\circ}$ of visual angle), a moderate amount (medium; $25 \%-35 \%$ of the screen; $6.64^{\circ}-9.38^{\circ}$ of visual angle), or a large amount (far; $40 \%-60 \%$ of the screen; $10.62^{\circ}-$ $15.94^{\circ}$ of visual angle) as shown in Figure 1 . We limited the placement of the pictures between $10 \%$ and $90 \%$ of the screen so that the images were never placed along the edge of the computer screen. For the different condition, one of the images was moved

Table 1. A summary of demographics, neuropsychological scores, and task performance per group

\begin{tabular}{lccc}
\hline & Young & $\begin{array}{c}\text { Aged (AU) } \\
\text { Unimpaired }\end{array}$ & $\begin{array}{c}\text { Aged (AI) } \\
\text { Impaired }\end{array}$ \\
\hline Years of age & $19.9(2.4)$ & $69.1(5.2)$ & $72.9(4.1)$ \\
Years of education & $14.1(1.7)^{\mathrm{a}}$ & $16.7(1.8)$ & $15.5(2.9)$ \\
Gender (male/female) & $3 \mathrm{M} / 17 \mathrm{~F}$ & $6 \mathrm{M} / 14 \mathrm{~F}$ & $5 \mathrm{M} / 5 \mathrm{~F}$ \\
RAVLT total performance & $53.5(6.7)$ & $56.2(6.4)$ & $43.4(6.1)^{\mathrm{b}}$ \\
$\begin{array}{l}\text { RAVLT immediate } \\
\text { performance }\end{array}$ & $12.1(1.9)$ & $12.2(1.5)$ & $8.3(1.9)^{\mathrm{b}}$ \\
$\begin{array}{l}\text { RAVLT delay } \\
\text { performance }\end{array}$ & $11.8(1.4)$ & $11.8(1.6)$ & $6.5(1.7)^{\mathrm{b}}$ \\
$\begin{array}{l}\text { Estimated IQ } \\
\text { Digit span performance }\end{array}$ & - & $120.8(5.5)$ & $115(6.7)^{\mathrm{b}}$ \\
$\begin{array}{l}\text { Mini-Mental State } \\
\text { examination }\end{array}$ & - & $18.9(4.5)$ & $17(3.8)$ \\
\hline
\end{tabular}

All data are reported as mean (SD).

${ }^{a}$ An unpaired $t$-test revealed higher years of education for the aged adults (16.3, SD 2.3) than the young adults (14.1, SD 1.7), $t_{(48)}=3.7, P<0.001$.

${ }^{\mathrm{b}}$ In addition, unpaired $t$-tests showed a poorer performance for the Al group relative to the AU group for RAVLT Total $t_{(28)}=5.2, P<0.0001$, RAVLT Immediate $t_{(28)}=6.3, P<0.0001$, and RAVLT Delay $t_{(28)}=8.6, P<0.0001$. Although there is a group difference in IQ $t_{(26)}=2.5, P<0.05$, these are largely overlapping distributions, and the Al group's IQ scores are certainly within normal limits. In addition, there was no relationship between IQ scores and performance on any of the tasks or other measures we used. in the $x$-coordinate by a percentage of the screen (i.e., $10 \%-20 \%$ in the close condition) and in the $y$-coordinate by a percentage of the screen (i.e., $10 \%-20 \%$ in the close condition), while the other image remained in its original location.

The probability to respond "different" for the SAME and three DIFFERENT (close, medium, and far) conditions for young and aged adults is shown in Figure 2A. A $2 \times 4$ analysis of variance (ANOVA) with group (young and aged adults) as a between-group factor and condition (same, close, medium, far) as a within-group factor revealed a main effect of condition, $F_{(3,192)}=35.62, P<$ 0.0001 . A post-hoc trend analysis revealed a positive linear trend across the four conditions, $r^{2}=0.62, P<0.0001$. There was no effect of group or an interaction, indicating no overall difference in spatial pattern separation ability between young and aged adults.

Since we were interested in the variability associated with healthy aging, we explored the aged group further. While rats are typically divided into impaired and unimpaired groups based on their performance in the Morris water maze (Gallagher et al. 1993), we divided the aged group into aged unimpaired (AU) and aged impaired (AI) based on their RAVLT delayed word learning performance. Importantly, the aged impaired individuals scored within the normal range for their own age group (ages 60-80). Aged unimpaired participants scored within the normal range for young individuals (ages 20-29) on the delayed test of the RAVLT (mean words recalled 11.8, range 9$15)$, whereas aged impaired individuals scored more than 1 standard deviation below these norms (mean words recalled 6.5, range $5-8)$. Thus, the aged impaired group was not clinically impaired and only mildly impaired relative to the young. While the aged unimpaired (69.1 yr, range 59-78) group is marginally different from the aged impaired (72.9 yr, range 67-80) group, $t_{(28)}=$ 2.02, $P=0.053$, there was not a significant correlation $\left(r^{2}=\right.$ 0.06 , ns) between performance on the DIFFERENT conditions and age as might be expected if age alone were responsible for the pattern separation impairments reported here. These data are presented in Figure 2B.

We entered the aged unimpaired and impaired groups into a $3 \times 4$ ANOVA with Group (Young, AI, and AU) and Condition as factors. We found a main effect of Condition as before, $F_{(3,188)}=$ 29.1, $P<0.0001$. Critically, we also found an effect of Group, $F_{(2,188)}=4.7, P<0.05$, such that the aged impaired group performed worse on the DIFFERENT conditions. We then calculated a separation bias score by averaging the three DIFFERENT conditions together (Fig. 2C) and analyzed these scores with a $2 \times 2$ ANOVA with Group and Condition as factors. Again, there was a main effect of Group $F_{(2,94)}=4.7, P<0.05$; a main effect of Condition, $F_{(1,94)}=500.8, P<0.0001 ;$ and an interaction, $F_{(2,94)}=4.7, P<0.05$. Bonferroni-corrected post-tests identified that the AI group was significantly impaired on the DIFFERENT trials compared to the AU group, $t_{(94)}=4.1, P<0.001$; and the Young group, $t_{(94)}=1.9, P<0.05$. These analyses all emphasize the same finding, namely, that AI individuals are impaired on the conditions taxing spatial pattern separation (i.e., DIFFERENT), but are not impaired on the condition that does not tax separation per se (i.e., SAME).

Using the RAVLT delayed recall performance to divide the aged group into $\mathrm{AI}$ and $\mathrm{AU}$ was an effective way to capture some of the individual variability in memory performance exhibited in the aged group. Indeed, there was a strong correlation between the aged impaired RAVLT scores and their performance on the DIFFERENT trials. We entered the RAVLT delayed recall scores into a linear regression with their performance on the average of the DIFFERENT trials and found a positive linear correlation such that as RAVLT delayed recall scores increased, performance on the different trials increased, $r^{2}=0.28, P<0.01$ (Fig. 3A). 


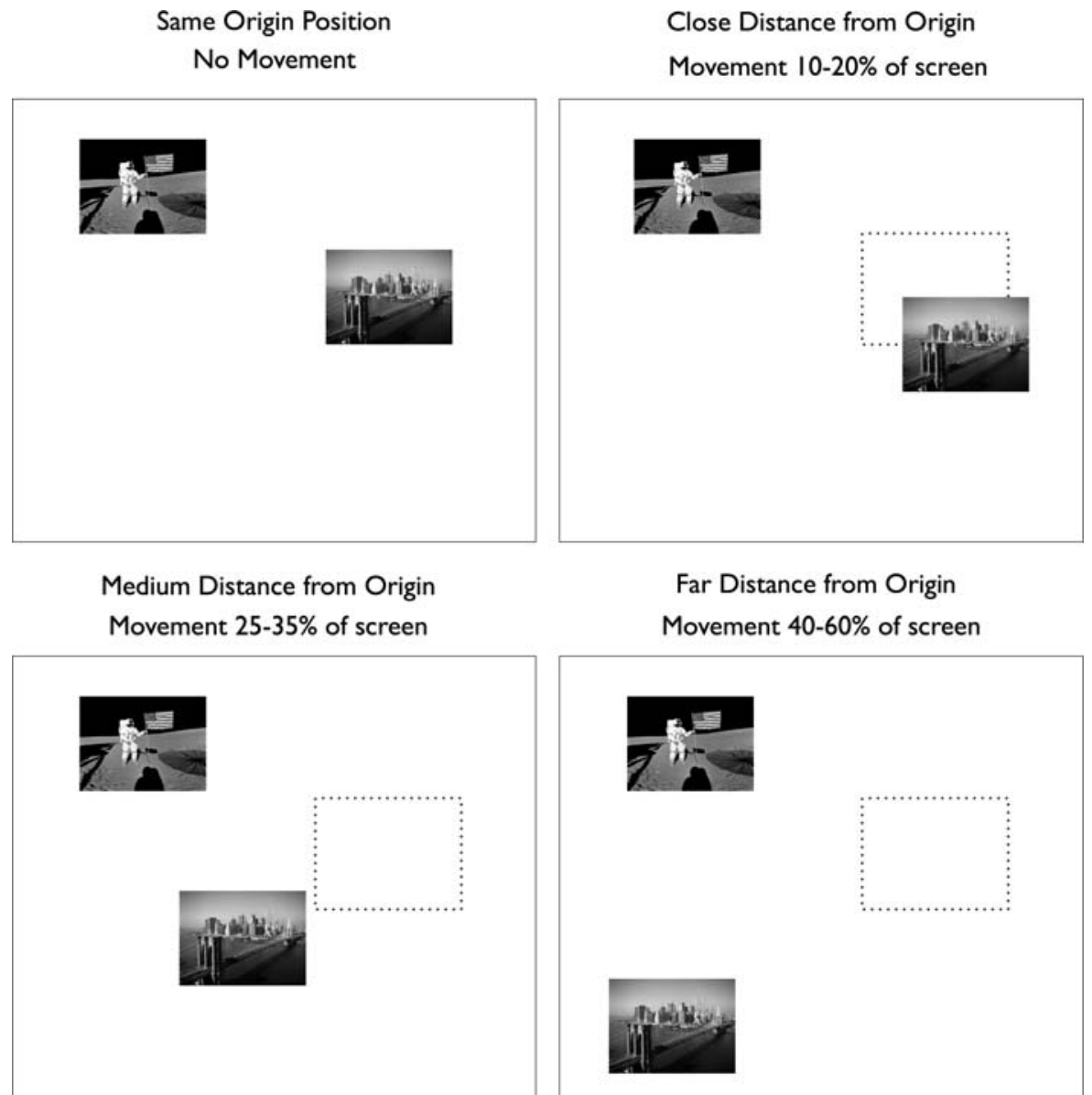

Figure 1. SAME and DIFFERENT (separated into close, medium, and far amounts of movement) conditions for the Spatial Paired Distance task. The dashed-line box demonstrates the original location of the second picture, but was not shown to the participants.

When the AI and AU groups were split, we observed that the AI group's correlation remained reliable, $r^{2}=0.40, P<0.05$, while the AU group no longer exhibited a significant correlation, $r^{2}=$ 0.03 , ns. These data support the notion that spatial pattern separation performance may be a sensitive index of memory variability in aging.

Since we collected additional behavioral measures on the same sample, we sought to determine if spatial pattern separation performance and RAVLT delayed recall performance might predict object pattern separation performance. Twenty-eight of the aged adults also participated in the object pattern separation paradigm as detailed by Yassa et al. (in press). Briefly, participants encoded everyday objects by simply making an indoor/outdoor judgment. During the test session, they viewed the same object (REPEATS), similar objects (LURES), and new objects (FOILS). They were required to make an "old," "similar," or "new" judgment for each item. We then computed a separation bias score by subtracting the probability of making "similar" responses to the FOILS from the probability of making "similar" responses to LURES, that is, $p$ ("similar") | LURE $-p$ ("similar") | FOIL. As reported by Yassa et al. (in press) the separation bias in the aging group was reduced relative to young adults, consistent with the Toner et al. (2009) findings.

If spatial pattern separation as assessed by the SPD task is engaging an underlying process similar to that engaged in object pattern separation, we would predict a positive relationship between SPD and object mnemonic similarity (OMS) task performance. We computed average performance on the DIFFERENT conditions (close, medium, and far) and entered it into a linear regression with the OMS separation score for each individual in the aged group. We observed a positive relationship, $r^{2}=0.26, P<$ 0.01 , as shown in Figure 3B. Likewise, we hypothesized a positive relationship between the OMS separation score and RAVLT delay performance, since we observed such a relationship with SPD performance. We entered these data into a linear regression and again observed a positive relationship, $r^{2}=0.22, P<0.05$ (with one OMS outlier greater than two SDs removed), as shown in Figure 3C. We also examined the relationship between SPD performance and Digit Span performance and estimated IQ in the aged group, but we did not find any significant correlations. These data indicate that these measures of both object and spatial pattern separation are behavioral manifestations of a similar underlying process that
A

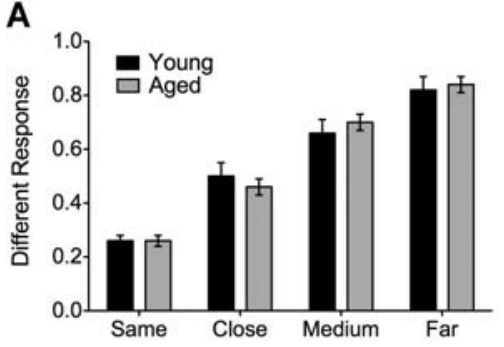

B

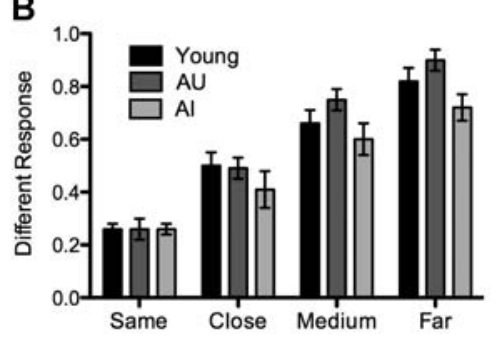

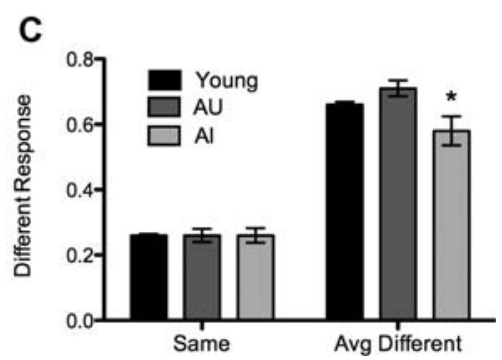

Figure 2. (A) The mean proportion correct for each of the four conditions. There is a main effect of Condition, with a linear trend of increasing DIFFERENT responses across the conditions, but no difference between the younger adults and aged adults. $(B)$ When the aged adults are separated according to their RAVLT Delay performance into impaired (AI) and unimpaired (AU), the Al adults perform significantly worse than both the young and the AU adults on the three DIFFERENT conditions. (C) Averaging the groups' performance on the DIFFERENT trials emphasizes the finding that Al performance is matched on the SAME condition and is selectively impaired on the DIFFERENT conditions that tax spatial pattern separation. 

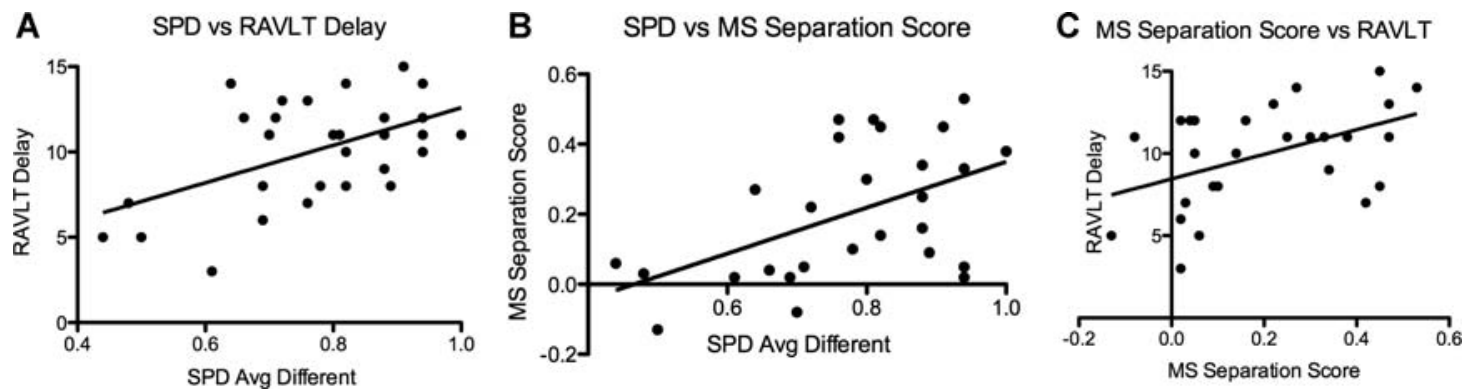

Figure 3. For the aged group, there is a positive relationship between SPD different trial performance and RAVLT delay performance ( $A$ ), SPD different trial performance and MS separation score performance $(B)$, and MS separation score performance and RAVLT delay performance $(C)$. These positive relationships indicate some shared underlying process that may be captured by individual differences in memory performance during the course of aging.

may also be somewhat accounted for by RAVLT delay recall performance.

One pertinent question is whether RAVLT performance would predict SPD performance in the Young group, making it a sensitive measure regardless of aging per se. When we examined this question by entering SPD performance on the average of the DIFFERENT conditions and RAVLT delay performance into a linear regression (two SPD outliers greater than two SDs removed), we found no evidence for this hypothesis $\left(r^{2}=0.02\right.$, ns). Likewise, it might appear circular to define the AI group based on their poorer RAVLT memory performance and then identify poorer performance on the SPD task. However, the AI versus AU difference is selective for the DIFFERENT condition, yet performance is matched for the SAME condition. One would expect both the SAME and DIFFERENT conditions to be similarly adversely affected if a general memory impairment could account for the poorer AI performance.

We suggest that these data support the notion of an impairment in spatial pattern separation processing in AI individuals. Ideally, we would have predicted a gradient of this effect, with more severe impairments in performance in the Close condition and matched performance in the Far condition. Unfortunately, the performance in the Close condition is near the floor, with all groups hovering around chance performance (50\%). This potential floor effect may be obscuring a greater deficit in the Close condition for the AI group. On the other end, performance on the Far condition may be suffering a bit from a ceiling effect. Performance in the Far condition is not much better than the same condition in any group, and that same performance is only $\sim 74 \%$ for each group. Therefore, the difficulty associated with this task may be such that we cannot create an "easy" enough Far condition to increase the percentage correct. Indeed, pilot testing on manipulations of this task (moving both items at test instead of just one, for example) did not result in greater accuracy performance for older or younger adults. While these data are not able to speak to a gradient of spatial pattern separation, we would argue that the selective impairments for the AI group for the DIFFERENT condition still reflect a deficit in spatial pattern separation processes.

The Spatial Paired Distance task presented here appears to be a measure that is sensitive to individual variations in memory performance associated with aging. The dentate gyrus seems a likely candidate for the source of this variability given its involvement in rodent (Small et al. 2004) and human aging studies (Small et al. 2002). Future research quantifying the structural and functional integrity of the dentate gyrus and other medial temporal lobe structures may elucidate those relationships with this task. Whether the variability associated with this task is a source of natural variation in the aged population or a precursor to mild cognitive impairment and possibly Alzheimer disease (AD) is also not clear. Longitudinal assessment of these or other individuals would be required to determine whether those in the AI group were more likely to develop $\mathrm{AD}$. If such is the case, then the SPD and object mnemonic similarity tasks may be particularly useful for early detection and diagnosis of pathological changes associated with dementia. Similarly, these tasks may be advantageous for use as outcome measures in clinical trials of new medications aimed at addressing these changes.

\section{Acknowledgments}

This research was supported by a University of California at Irvine research fund awarded to C.E.L.S. and in part by a grant from the National Institutes on Aging (R03 AG032015). We thank Samantha Rutledge and Megan Deaton for their assistance in data collection.

\section{References}

Bakker A, Kirwan CB, Miller NI, Stark CEL. 2008. Pattern separation in the human hippocampal CA3 and dentate gyrus. Science 319: 1640-1642.

Cravens CJ, Vargas-Pinto N, Christian KM, Nakazawa K. 2006. CA3 NMDA receptors are crucial for rapid and automatic representation of context memory. Eur J Neurosci 24: 1771-1780.

Folstein MF, Folstein SE, McHugh PR. 1975. 'Mini-mental state.' A practical method for grading the cognitive state of patients for the clinician. $J$ Psychiatr Res 12: 189-198.

Gallagher M, Burwell R, Burchinal M. 1993. Severity of spatial learning impairment in aging: Development of a learning index for performance in the Morris water maze. Behav Neurosci 107: 618-626.

Gallagher M, Colantuoni C, Eichenbaum H, Haberman R, Rapp PR, Tanila H, Wilson IA. 2006. Individual differences in neurocognitive aging of the medial temporal lobe. Age 28: 221-233.

Gilbert PE, Kesner RP. 2006. The role of the dorsal CA3 hippocampal subregion in spatial working memory and pattern separation. Behav Brain Res 169: 142-149.

Gilbert PE, Kesner RP, Lee I. 2001. Dissociating hippocampal subregions: A double dissociation between dentate gyrus and CA1. Hippocampus 11: 626-636.

Goodrich-Hunsaker NJ, Hunsaker MR, Kesner RP. 2008. The interactions and dissociations of the dorsal hippocampus subregions: How the dentate gyrus, CA3, and CA1 process spatial information. Behav Neurosci 122: 16-26.

Hilborn JV, Strauss E, Hultsch DF, Hunter MA. 2009. Intraindividual variability across cognitive domains: Investigation of dispersion levels and performance profiles in older adults. J Clin Exp Neuropsychol 31: 412-424.

Hunsaker MR, Rosenberg JS, Kesner RP. 2008. The role of the dentate gyrus, CA3a,b, and CA3c for detecting spatial and environmental novelty. Hippocampus 18: 1064-1073.

Kesner RP, Lee I, Gilbert P. 2004. A behavioral assessment of hippocampal function based on a subregional analysis. Rev Neurosci 15: 333-351.

Kirwan CB, Stark CEL. 2007. Overcoming interference: An fMRI investigation of pattern separation in the medial temporal lobe. Learn Mem 14: $625-633$. 
Kubik S, Miyashita T, Guzowski JF. 2007. Using immediate-early genes to map hippocampal subregional functions. Learn Mem 14: 758-770.

Lee HK, Min SS, Gallagher M, Kirkwood A. 2005. NMDA receptorindependent long-term depression correlates with successful aging in rats. Nat Neurosci 8: $1657-1659$.

Leutgeb S, Leutgeb JK. 2007. Pattern separation, pattern completion, and new neuronal codes within a continuous CA3 map. Learn Mem 14: $745-757$.

Leutgeb S, Leutgeb JK, Treves A, Moser MB, Moser EI. 2004. Distinct ensemble codes in hippocampal areas CA3 and CA1. Science 305: $1295-1298$.

Leutgeb S, Leutgeb JK, Barnes CA, Moser EI, McNaughton BL, Moser MB. 2005. Independent codes for spatial and episodic memory in hippocampal neuronal ensembles. Science 309: 619-623.

Leutgeb JK, Leutgeb S, Moser MB, Moser EI. 2007. Pattern separation in the dentate gyrus and CA3 of the hippocampus. Science 315: 961-966

McClelland JL, McNaughton BL, O'Reilly RC. 1995. Why there are complementary learning systems in the hippocampus and neocortex: Insights from the successes and failures of connectionist models of learning and memory. Psychol Rev 102: 419-457.

McHugh SB, Niewoehner B, Rawlins JNP, Bannerman DM. 2008. Dorsal hippocampal $N$-methyl-D-aspartate receptors underlie spatial working memory performance during non-matching to place testing on the T-maze. Behav Brain Res 186: 41-47.

Norman KA, O'Reilly RC. 2003. Modeling hippocampal and neocortical contributions to recognition memory: A complementary-learningsystems approach. Psych Review 110: 611-646.

Rey A. 1941. L'examen psychologique dans les cas d'encephalopathie traumatique. Arch Psychol 28: 286-340.

Robitsek RJ, Fortin NJ, Koh MT, Gallagher M, Eichenbaum H. 2008. Cognitive aging: A common decline of episodic recollection and spatial memory in rats. J Neurosci 28: 8945-8954.

Scheff SW, Price DA, Schmitt FA, Mufson EJ. 2006. Hippocampal synaptic loss in early Alzheimer's disease and mild cognitive impairment. Neurobiol Aging 27: 1372-1384.
Schoenberg MR, Duff K, Scott JG, Adams RL. 2003. An evaluation of the clinical utility of the OPIE-3 as an estimate of premorbid WAIS-III FSIQ. Clin Neuropsychol 17: 308-321.

Small SA, Tsai WY, DeLaPaz R, Mayeux R, Stern Y. 2002. Imaging hippocampal function across the human life span: Is memory decline normal or not? Ann Neurol 51: 290-295.

Small SA, Chawla MK, Buonocore M, Rapp PR, Barnes CA. 2004. Imaging correlates of brain function in monkeys and rats isolates a hippocampal subregion differentially vulnerable to aging. Proc Natl Acad Sci 101: 7181-7186.

Smith TD, Adams MM, Gallagher M, Morrison JH, Rapp PR. 2000. Circuit-specific alterations in hippocampal synaptophysin immunoreactivity predict spatial learning impairment in aged rats. I Neurosci 20: 6587-6593.

Toner CK, Pirogovsky E, Kirwan CB, Gilbert PE. 2009. Visual object pattern separation deficits in nondemented older adults. Learn Mem 16: $338-342$.

Treves A, Rolls ET. 1994. Computational analysis of the role of the hippocampus in memory. Hippocampus 4: 374-391.

Vazdarjanova A, Guzowski JF. 2004. Differences in hippocampal neurona population responses to modifications of an environmental context: Evidence for distinct, yet complementary, functions of CA3 and CA1 ensembles. J Neurosci 24: 6489-6496.

Wechsler D. 1997. Wechsler Adult Intelligence Scale (WAIS-III): Administration and scoring manual. The Psychological Corporation, San Antonio, TX.

Wilson IA, Gallagher M, Eichenbaum H, Tanila H. 2006. Neurocognitive aging: Prior memories hinder new hippocampal encoding. Trends Neurosci 29: 662-670.

Yassa M, Lacy J, Stark S, Albert MS, Gallagher M, Stark CEL. 2010. Pattern separation deficits associated with increased hippocampal CA3 and dentate gyrus activity in nondemented older adults. Hippocampus (in press).

Received February 1, 2010; accepted in revised form April 14, 2010. 


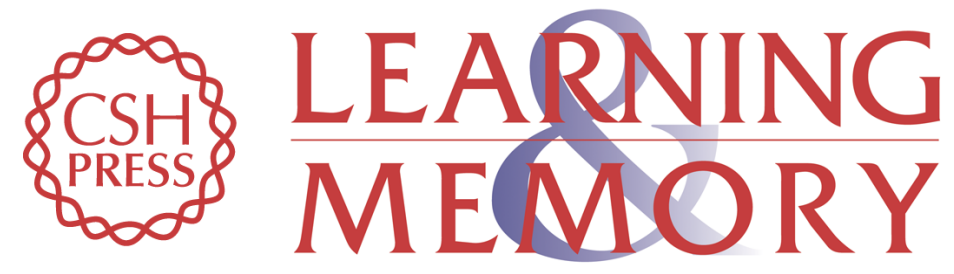

\section{Individual differences in spatial pattern separation performance associated with healthy aging in humans}

Shauna M. Stark, Michael A. Yassa and Craig E.L. Stark

Learn. Mem. 2010, 17:

Access the most recent version at doi:10.1101//m.1768110

References This article cites 32 articles, 12 of which can be accessed free at: http://learnmem.cshlp.org/content/17/6/284.full.html\#ref-list-1

License

Email Alerting Receive free email alerts when new articles cite this article - sign up in the box at the Service top right corner of the article or click here. 